\title{
Bis-(2-amino-5-selenazoyl) Ketone as a Superoxide Anion-Scavenger
}

\author{
Akihiro Sekiguchi, ${ }^{a}$ Atsuyoshi Nishina, ${ }^{a}$ Hirokazu Kimura, ${ }^{b}$ Ryo-hei Fukumoto, ${ }^{a}$ \\ Masakazu Kogami ${ }^{c}$ Hideharu Ishinara, ${ }^{c}$ and Mamoru KoKetsu ${ }^{*}, d$ \\ ${ }^{a}$ Gunma Industrial Technology Center; 884-1 Kamesato, Maebashi, Gunma 379-2147, Japan: ${ }^{b}$ Gunma Prefectural \\ Institute of Public Health and Environmental Sciences; 378 Kamioki, Maebashi, Gunma 371-0052, Japan: ${ }^{c}$ Department of \\ Chemistry, Faculty of Engineering, Gifu University; and ${ }^{d}$ Division of Instrumental Analysis, Life Science Research Center, \\ Gifu University; Gifu 501-1193, Japan. \\ Received February 14, 2006; accepted April 13, 2006; published online April 18, 2006
}

\begin{abstract}
We investigated the superoxide anion scavenging effects of 2-amino-1,3- selenazoles and bis-(2-amino-5-selenazoyl) ketones using a highly sensitive quantitative chemiluminescence method. At $166 \mu \mathrm{M}$, the 2-amino-1,3-selenazoles and bis-(2-amino-5-selenazoyl) ketones scavenged in the range of 10.0 to $80.0 \%$ of $\mathrm{O}_{2}^{-}$. Bis[2-dimethylamino-5-(1,3-selenazolyl)] ketone exhibited the strongest superoxide anion-scavenging activity among the six kinds of 2-amino-1,3-selenazoles and three kinds of bis-(2-amino-5-selenazoyl) ketones. The 50\% inhibitory concentration $\left(\mathrm{IC}_{50}\right)$ of bis[2-dimethylamino-5-(1,3-selenazolyl)] ketone was determined to be $37.1 \mu_{\mathrm{M}}$. Thus, bis[2dimethylamino-5-(1,3-selenazolyl)] ketone acted in vitro as effective and potentially useful $\mathrm{O}_{2}^{-}$scavenger.
\end{abstract}

Key words 2-amino-1,3-selenazole; heterocycle; superoxide radical; scavenging effect; superoxide anion-scavenging activities (SOSAs)

Accumulating evidence suggests that even under normal physiological conditions, reactive oxygen species (ROSs) are generated in aerobic cells, and therefore, exposure to ROSs is inevitable. ${ }^{1)}$ ROSs also react with biomacromolecules, including DNA and proteins, leading to the degeneration of these molecules. ${ }^{2,3)}$ In addition, phagocytes (granulocytes, monocytes/macrophages, eosinophils, and basophils) generate large amounts of ROSs, leading to cell/tissue injuries due to excessive inflammation. ${ }^{4}$-6) In extreme instances such as endotoxin shock, neutrophils kill the infected host. ${ }^{7}$

Antioxidative enzymes and compounds such as superoxide dismutases (SODs), catalase, glutathione peroxidase (GPX), and some vitamins act as antioxidants. ${ }^{4,6}$ GPX is an important antioxidant enzyme and effectively eliminates $\mathrm{H}_{2} \mathrm{O}_{2}$ in vitro and in vivo ${ }^{6,8)}$ GPX essentially contains selenium atoms in active domain. ${ }^{8)}$ Various studies have reported that selenoproteins including GPX reduce oxidative stress in cells. ${ }^{9,10)}$ Thus, various organic selenium compounds may be a candidate of ROSs scavenger.

The ebselen, a five-membered ring selenium-containing heterocyclic compound showing glutathione peroxidaselike activity, ${ }^{11)}$ is a synthetic antioxidant due to a ROSs scavenger. ${ }^{12)}$ The antioxidative effect of the ebselen is selective blockade of leukocyte infiltration and activation, resulting in reduction of the $\mathrm{H}_{2} \mathrm{O}_{2}$ level. ${ }^{12)}$ This compound is a multifunctional antioxidant and a potential chemopreventive agent in inflammation-associated carcinogenesis. ${ }^{13)}$ Thus, various types of organic selenium compounds may be applicable to reduce oxidative stres. ${ }^{14-16)}$

We have synthesized various organic selenium compounds including selenoamides,${ }^{14)}$ selenoureas, thioureas, ${ }^{15)}$ and selenocarbamates. ${ }^{16)}$ Moreover, most of theses compounds have also effectively scavenged $\mathrm{O}_{2}^{-}$in vitro. ${ }^{15,16)}$ In this study, superoxide anion-scavenging activities (SOSAs) of a newly synthesized series of five-membered ring selenium-containing heterocyclic compounds, 2-amino-1,3- selenazoles and bis-(2-amino-5-selenazoyl) ketones were tested in vitro.

\section{MATERIALS AND METHODS}

Materials 2-Amino-1,3-selenazoles and bis-(2-amino-5selenazoyl) ketones were prepared according to procedures previously reported. ${ }^{17)}$ A Cypridina luciferin analogue, 2methyl-6-(4-methoxyphenyl)-3,7-dihydroimidazo-[1,2-a]pyrazin-3-one hydrochloride (MCLA) was obtained from Tokyo Kasei (Tokyo, Japan) for a use as a chemiluminescent probe for superoxide radicals. MCLA was dissolved in doubly distilled water and stored at $-80^{\circ} \mathrm{C}$ prior to usage. The concentration of MCLA solution was determined by absorbance at $430 \mathrm{~nm}$ using an absorbance coefficient value of $\varepsilon=9600 \mathrm{M}^{-1} \mathrm{~cm}^{-1}$, as previously described. ${ }^{18)} \mathrm{SOD}$ (Lyophilized powder, 3400 units/mg protein) and xanthine oxidase (XOD grade III) were purchased from Sigma Chemical (St. Louis, MO, U.S.A.). Hypoxanthine was purchased from Wako Pure Chemical (Osaka, Japan) and used without further purification. All other chemicals and solvents were analytical grade and used without further purification.

Synthetic Methods for the Preparation of 2-Amino-1,3selenazoles 1 and Bis-(2-amino-5-selenazoyl) Ketones 2 5-Acetyl-2-dimethylamino-1,3-selenazole 1a: Chloroacetone ( $24 \mu \mathrm{l}, 0.3 \mathrm{mmol})$ was added to stirred solution of $N, N$-dimethyl- $N N^{\prime}$-(dimethylselenocarbamoyl)formamidine $\quad(0.62 \mathrm{~g}$, $0.3 \mathrm{mmol})$ in dry methanol $(3 \mathrm{ml})$ under an argon atmosphere. The reaction mixture was refluxed for $2 \mathrm{~h}$. The mixture was extracted with dichloromethane. The extract was evaporated to dryness. The residue was purified by flash chromatography on silica gel with ethyl acetate-hexane $(1: 2)$ to give 1a $(64 \mathrm{mg}, 98 \%)$ as pale red crystals. $\mathrm{mp}$ : $100-102^{\circ} \mathrm{C}$. IR (KBr): $1622 \mathrm{~cm}^{-1} \cdot{ }^{1} \mathrm{H}-\mathrm{NMR}(500 \mathrm{MHz}$, $\mathrm{CDCl}_{3}, \mathrm{rt}$ ): $\delta=2.44\left(\mathrm{~s}, 3 \mathrm{H}, \mathrm{CH}_{3}\right), 3.19$ (br s, $6 \mathrm{H}, \mathrm{N}-\mathrm{CH}_{3}$ ), $7.78(\mathrm{~s}, 1 \mathrm{H}, \mathrm{CH}) .{ }^{1} \mathrm{H}-\mathrm{NMR}\left(500 \mathrm{MHz}, \mathrm{CDCl}_{3},-40^{\circ} \mathrm{C}\right)$ : $\delta=2.49\left(\mathrm{~s}, 3 \mathrm{H}, \mathrm{CH}_{3}\right), 3.14\left(\right.$ br s, $\left.3 \mathrm{H}, \mathrm{N}-\mathrm{CH}_{3}\right), 3.33($ br s, $3 \mathrm{H}$, $\left.\mathrm{N}-\mathrm{CH}_{3}\right), 7.83(\mathrm{~s}, 1 \mathrm{H}, \mathrm{CH}) \cdot{ }^{13} \mathrm{C}-\mathrm{NMR}\left(125 \mathrm{MHz}, \mathrm{CDCl}_{3}\right.$, $\mathrm{rt}): \delta=25.3,41.2,134.2,149.6,178.4,190.3 .{ }^{13} \mathrm{C}-\mathrm{NMR}$ $\left(125 \mathrm{MHz}, \mathrm{CDCl}_{3},-40^{\circ} \mathrm{C}\right): \delta=25.4,39.1,43.7,133.5$, $149.9,178.2,190.8 .{ }^{77} \mathrm{Se}-\mathrm{NMR}\left(95 \mathrm{MHz}, \mathrm{CDCl}_{3}\right): \delta=523.1$. MS (FAB): $m / z=219\left[\mathrm{M}^{+}+1\right]$. Anal. Calcd for $\mathrm{C}_{7} \mathrm{H}_{10} \mathrm{~N}_{2} \mathrm{OSe}$ : 
C, 38.72; H, 4.64; N, 12.90. Found; C, 38.99; H, 4.95; N, 12.63 .

5-Acetyl-2-piperidino-1,3-selenazole 1b: Yield 93\%. White crystals. mp: $74-76^{\circ} \mathrm{C}$. IR $(\mathrm{KBr}): 1624 \mathrm{~cm}^{-1} \cdot{ }^{1} \mathrm{H}-$ NMR $\left(500 \mathrm{MHz}, \mathrm{CDCl}_{3}, \mathrm{rt}\right): \delta=1.70\left(\mathrm{~m}, 6 \mathrm{H}, \mathrm{CH}_{2}\right), 2.43(\mathrm{~s}$, $\left.3 \mathrm{H}, \mathrm{CH}_{3}\right), 3.57\left(\mathrm{~m}, 4 \mathrm{H}, \mathrm{CH}_{2}\right), 7.75(\mathrm{~s}, 1 \mathrm{H}, \mathrm{CH}) .{ }^{13} \mathrm{C}-\mathrm{NMR}$ $\left(125 \mathrm{MHz}, \mathrm{CDCl}_{3}, \mathrm{rt}\right): \delta=23.9,25.2,25.3,51.0,133.1$, 149.7, 178.1, 190.3. ${ }^{77} \mathrm{Se}-\mathrm{NMR}\left(95 \mathrm{MHz}, \mathrm{CDCl}_{3}\right): \delta=$ 522.4. MS (FAB): $m / z=259\left[\mathrm{M}^{+}+1\right]$. Anal. Calcd for $\mathrm{C}_{10} \mathrm{H}_{14} \mathrm{~N}_{2} \mathrm{OSe}$ : C, 46.70; H, 5.49; N, 10.89. Found; C, 46.88; $\mathrm{H}, 5.51 ; \mathrm{N}, 10.70$.

5-Acetyl-2-morpholino-1,3-selenazole 1c: Yield 74\%. Pale yellow crystals. mp: $134-135^{\circ} \mathrm{C}$. IR (KBr): 1636 $\mathrm{cm}^{-1} .{ }^{1} \mathrm{H}-\mathrm{NMR}\left(500 \mathrm{MHz}, \mathrm{CDCl}_{3}, \mathrm{rt}\right): \delta=2.45\left(\mathrm{~s}, 3 \mathrm{H}, \mathrm{CH}_{3}\right)$, $3.59\left(\mathrm{t}, J=5.2 \mathrm{~Hz}, 4 \mathrm{H}, \mathrm{CH}_{2}\right), 3.81\left(\mathrm{t}, J=5.2 \mathrm{~Hz}, 4 \mathrm{H}, \mathrm{CH}_{2}\right)$, 7.77 (s, $1 \mathrm{H}, \mathrm{CH}) .{ }^{13} \mathrm{C}-\mathrm{NMR}\left(125 \mathrm{MHz}, \mathrm{CDCl}_{3}, \mathrm{rt}\right): \delta=$ 25.4, 49.5, 65.9, 134.6, 149.0, 178.6, 190.4. ${ }^{77}$ Se-NMR $\left(95 \mathrm{MHz}, \mathrm{CDCl}_{3}\right): \delta=532.4$. $\mathrm{MS}(\mathrm{FAB}): m / z=261\left[\mathrm{M}^{+}+1\right]$.

5-Benzoyl-2-dimethylamino-1,3-selenazole 1d: Yield quantitatively. Yellow crystals. $\mathrm{mp}: 69-70{ }^{\circ} \mathrm{C}$. IR $(\mathrm{KBr})$ : $1603 \mathrm{~cm}^{-1}$. ${ }^{1} \mathrm{H}-\mathrm{NMR}\left(500 \mathrm{MHz}, \mathrm{CDCl}_{3}, \mathrm{rt}\right): \delta=3.22$ (br s, $\left.6 \mathrm{H}, \mathrm{N}-\mathrm{CH}_{3}\right), 7.43-7.46(\mathrm{~m}, 2 \mathrm{H}, \mathrm{Ar}), 7.50-7.53(\mathrm{~m}, 1 \mathrm{H}$, Ar), $7.68(\mathrm{~s}, 1 \mathrm{H}, \mathrm{CH}), 7.73-7.75(\mathrm{~m}, 2 \mathrm{H}, \mathrm{Ar}) .{ }^{13} \mathrm{C}-\mathrm{NMR}$ $(125 \mathrm{MHz}, \mathrm{CDCl} 3, \mathrm{rt}): \delta=128.5,128.7,131.6,134.0,138.6$, 152.3, 178.4, 188.3. ${ }^{13} \mathrm{C}-\mathrm{NMR}\left(125 \mathrm{MHz}, \mathrm{CDCl}_{3},-40^{\circ} \mathrm{C}\right)$ : $\delta=39.0,43.6,128.2,128.4,131.4,133.0,137.8,152.5$, 177.9, 188.2. ${ }^{77} \mathrm{Se}-\mathrm{NMR}\left(95 \mathrm{MHz}, \mathrm{CDCl}_{3}\right): \delta=533.8 . \mathrm{MS}$ (CI): $m / z=281\left[\mathrm{M}^{+}+1\right]$. Anal. Calcd for $\mathrm{C}_{12} \mathrm{H}_{12} \mathrm{~N}_{2} \mathrm{OSe}: \mathrm{C}$, 51.62; H, 4.33; N, 10.03. Found; C, 51.57; H, 4.65; N, 9.93.

5-Benzoyl-2-piperidino-1,3-selenazole 1e: Yield quantitatively. Light yellow crystals. mp: $123-125^{\circ} \mathrm{C}$. IR $(\mathrm{KBr})$ : $1611 \mathrm{~cm}^{-1} .{ }^{1} \mathrm{H}-\mathrm{NMR}\left(500 \mathrm{MHz}, \mathrm{CDCl}_{3}, \mathrm{rt}\right): \delta=1.71(\mathrm{~m}, 6 \mathrm{H}$, $\left.\mathrm{CH}_{2}\right), 3.60\left(\mathrm{~m}, 4 \mathrm{H}, \mathrm{CH}_{2}\right), 7.44-7.47$ (m, 2H, Ar), 7.51$7.54(\mathrm{~m}, 1 \mathrm{H}, \mathrm{Ar}), 7.65(\mathrm{~s}, 1 \mathrm{H}, \mathrm{CH}), 7.74-7.76(\mathrm{~m}, 2 \mathrm{H}, \mathrm{Ar})$. ${ }^{13} \mathrm{C}-\mathrm{NMR}\left(125 \mathrm{MHz}, \mathrm{CDCl}_{3}, \mathrm{rt}\right): \delta=24.0,25.2,51.1,128.3$, $128.5,131.4,132.9,138.6,152.3,178.0,188.2 .{ }^{77} \mathrm{Se}-\mathrm{NMR}$ $\left(95 \mathrm{MHz}, \mathrm{CDCl}_{3}\right): \delta=532.8 \mathrm{MS}(\mathrm{CI}): m / z=321\left[\mathrm{M}^{+}+1\right]$.

5-Benzoyl-2-morpholino-1,3-selenazole 1f: Yield quantitatively. White crystals. mp: $130-132{ }^{\circ} \mathrm{C}$. IR (KBr): 1612 $\mathrm{cm}^{-1} .{ }^{1} \mathrm{H}-\mathrm{NMR} \quad\left(500 \mathrm{MHz}, \mathrm{CDCl}_{3}, \mathrm{rt}\right): \delta=3.63$ (t, $J=$ $\left.5.2 \mathrm{~Hz}, 4 \mathrm{H}, \mathrm{CH}_{2}\right), 3.82\left(\mathrm{t}, J=5.2 \mathrm{~Hz}, 4 \mathrm{H}, \mathrm{CH}_{2}\right), 7.45-7.48$ $(\mathrm{m}, 2 \mathrm{H}, \mathrm{Ar}), 7.53-7.56(\mathrm{~m}, 1 \mathrm{H}, \mathrm{Ar}), 7.68(\mathrm{~s}, 1 \mathrm{H}, \mathrm{CH})$, $7.75-7.77$ (m, 2H, Ar). ${ }^{13} \mathrm{C}-\mathrm{NMR}\left(125 \mathrm{MHz} \mathrm{CDCl}_{3}, \mathrm{rt}\right)$ : $\delta=49.6,66.0,128.4,128.6,131.6,134.3,138.3,151.5$, 178.5, 188.3. ${ }^{77} \mathrm{Se}-\mathrm{NMR}\left(95 \mathrm{MHz}, \mathrm{CDCl}_{3}\right): \delta=543.5$. MS (CI): $m / z=323\left[\mathrm{M}^{+}+1\right]$.

Bis[2-dimethylamino-5-(1,3-selenazolyl)] Ketone 2a: 1,3Dichloroacetone $(13 \mathrm{mg}, 0.1 \mathrm{mmol})$ was added to stirred solution of $N, N$-dimethyl- $N^{\prime}$-(dimethylselenocarbamoyl)formamidine (42 mg, $0.2 \mathrm{mmol}$ ) in dry acetonitrile $(3 \mathrm{ml})$ under an argon atmosphere. The reaction mixture was stirred at $50^{\circ} \mathrm{C}$ for $1 \mathrm{~h}$. The mixture was cooled off until $25^{\circ} \mathrm{C}$. Then triethylamine $(0.10 \mathrm{ml}, 0.7 \mathrm{mmol})$ was added into the mixture. The reaction mixture was stirred for $30 \mathrm{~min}$. at $25^{\circ} \mathrm{C}$. The mixture extracted with dichloromethane. The extract was concentrated to dryness. The residue was purified by flash chromatography on silica gel with ethyl acetate-hexane $(1: 1)$ to give $\mathbf{2 a}(33 \mathrm{mg}, 87 \%)$ as orange crystals. mp: $225-227^{\circ} \mathrm{C}$. IR (KBr): $1572 \mathrm{~cm}^{-1}$. UV/VIS $\left(\mathrm{CHCl}_{3}\right) \lambda_{\max }(\log \varepsilon): 392$ (2.74). ${ }^{1} \mathrm{H}-\mathrm{NMR}\left(500 \mathrm{MHz}, \mathrm{CDCl}_{3}, \mathrm{rt}\right): \delta=3.20$ (br s, $12 \mathrm{H}$, $\left.\mathrm{N}-\mathrm{CH}_{3}\right), 7.89$ (s, 2H, CH). ${ }^{13} \mathrm{C}-\mathrm{NMR}\left(125 \mathrm{MHz} \mathrm{CDCl}_{3}, \mathrm{rt}\right)$ : $\delta=41.2,132.8,147.1,177.1,178.5 .{ }^{77} \mathrm{Se}-\mathrm{NMR}(95 \mathrm{MHz}$, $\mathrm{CDCl}_{3}$ ): $\delta=545.4$. MS (FAB): $m / z=379\left[\mathrm{M}^{+}+1\right]$.

Bis[2-piperidino-5-(1,3-selenazolyl)] Ketone 2b: Yield 91\%. Orange crystals. mp: $260-262^{\circ} \mathrm{C}$. IR (KBr): 1541 $\mathrm{cm}^{-1}$. UV/VIS $\left(\mathrm{CHCl}_{3}\right) \lambda_{\max }(\log \varepsilon): 399$ (3.22). ${ }^{1} \mathrm{H}-\mathrm{NMR}$ $\left(500 \mathrm{MHz}, \mathrm{CDCl}_{3}, \mathrm{rt}\right): \delta=1.71\left(\mathrm{~m}, 12 \mathrm{H}, \mathrm{CH}_{2}\right), 3.57(\mathrm{~m}$, $\left.8 \mathrm{H}, \mathrm{CH}_{2}\right), 7.84(\mathrm{~s}, 2 \mathrm{H}, \mathrm{CH}) .{ }^{13} \mathrm{C}-\mathrm{NMR}\left(125 \mathrm{MHz}, \mathrm{CDCl}_{3}\right.$, $\mathrm{rt}): \delta=24.1,25.3,51.0,132.1,147.2,177.1,178.5 .{ }^{77} \mathrm{Se}-$ NMR $\left(95 \mathrm{MHz}, \mathrm{CDCl}_{3}\right): \delta=545.5$. MS (FAB): $m / z=$ $459\left[\mathrm{M}^{+}+1\right]$.

Bis[2-morpholino-5-(1,3-selenazolyl)] Ketone 2c: Yield $82 \%$. Orange crystals. mp: $262-263{ }^{\circ} \mathrm{C}$. IR (KBr): 1554 $\mathrm{cm}^{-1}$. UV/VIS $\left(\mathrm{CHCl}_{3}\right) \lambda_{\max }(\log \varepsilon): 391$ (3.20). ${ }^{1} \mathrm{H}-\mathrm{NMR}$ $\left(500 \mathrm{MHz}, \mathrm{CDCl}_{3}, \mathrm{rt}\right): \delta=3.59\left(\mathrm{t}, J=5.2 \mathrm{~Hz}, 8 \mathrm{H}, \mathrm{CH}_{2}\right)$, $3.82\left(\mathrm{t}, J=5.2 \mathrm{~Hz}, 8 \mathrm{H}, \mathrm{CH}_{2}\right), 7.87(\mathrm{~s}, 2 \mathrm{H}, \mathrm{CH}) .{ }^{13} \mathrm{C}-\mathrm{NMR}$ $\left(125 \mathrm{MHz} \mathrm{CDCl}_{3}, \mathrm{rt}\right): \delta=49.5,66.1,133.2,146.9,177.7$, 178.7. ${ }^{77} \mathrm{Se}-\mathrm{NMR}\left(95 \mathrm{MHz}, \mathrm{CDCl}_{3}\right): \delta=555.0$. MS (FAB): $m / z=463\left[\mathrm{M}^{+}+1\right]$. Anal. Calcd for $\mathrm{C}_{15} \mathrm{H}_{18} \mathrm{~N}_{4} \mathrm{O}_{3} \mathrm{Se}_{2}: \mathrm{C}$, 39.14; H, 3.94; N, 12.17. Found; C, 38.86; H, 4.28; N, 11.97.

Assay of Superoxide Anion-Scavenging Activities (SOSAs) The SOSAs of 2-amino-1,3-selenazoles $\mathbf{1}$ and bis-(2-amino-5-selenazoyl) ketones 2 were measured by a previously reported method. ${ }^{18)}$ In brief, the reaction mixture contained $5.8 \times 10^{-7} \mathrm{M}$ MCLA, $5 \times 10^{-5} \mathrm{M}$ hypoxanthine, xanthine oxidase $(6.5 \mathrm{U})$, and $50 \mathrm{~mm}$ Tris- $\mathrm{HCl}$ buffer containing $0.1 \mathrm{~mm}$ EDTA at $\mathrm{pH} 7.8$, in the presence or absence of various concentrations of compounds $\mathbf{1}$ and 2. To evaluate certain SOSAs, we added SOD standard solutions (0.6 to $30 \mathrm{ng} / \mathrm{ml}$ ) to the reaction mixture instead of compounds 1 and 2. Total volume was $3.0 \mathrm{ml}$. Compounds 1 and $\mathbf{2}(25 \mathrm{~mm})$ were dissolved in DMSO and stored at $-80{ }^{\circ} \mathrm{C}$ prior to use. Chemiluminescence was measured using a luminometer (Aloka, BLR201) at $25^{\circ} \mathrm{C}$. Chemiluminescence measurement was initiated by the addition of 2-methyl-6-(4methoxyphenyl)-3,7-dihydroimidazo[1,2-a]pyrazin-3-one hydrochloride (MCLA) to the standard incubation mixture excluding XOD, continued for 2 min without XOD and for an additional 2 min after addition of XOD. A representative example of a measurement of the effect of $\mathbf{2 a}$ on MCLA-dependent luminescence is shown in Fig. 1. When the compounds had strong SOSAS at $166 \mu \mathrm{M}$, we also measured at $4.17,16.7,41.7$ and $83.3 \mu \mathrm{M}$. Percentage of inhibition of MCLA dependent chemiluminescence was calculated as a previously described. ${ }^{18)}$ Inhibition concentration of $50 \%$ $\left(\mathrm{IC}_{50}\right)$ was calculated by six concentrations of $\mathbf{2 a}(4.17,16.7$, $27.7,41.7,83.3$ and $166 \mu \mathrm{M})$. In this study, it was measured twice about the same sample and average value was used.

\section{RESULTS AND DISCUSSION}

Structures of these 2-amino-1,3-selenazoles 1 and bis-[2dimethylamino-5-(1,3-selenazolyl)] ketones $\mathbf{2}$ are shown in Table 1, where the SOSAs of the compounds also are summarized (Table1). As results, bis-[5-(1,3-selenazolyl)] ketones 2 showed stronger activities than 1,3-selenazoles $\mathbf{1}$. Among them, bis[2-dimethylamino-5-(1,3-selenazolyl)] ketone $\mathbf{2} \mathbf{a}$ and bis[2-morpholino-5-(1,3-selenazolyl)] ketone $\mathbf{2 c}$ had the highest SOSAs at $166 \mu \mathrm{M}(80.0 \%$ and $68.6 \%$, respectively). The effect of $\mathbf{2 a}$ was dose dependent (Fig. 1). Compound 2a was sufficiently active to suggest further testing, by serial dilutions, $50 \%$ inhibitory concentrations $\left(\mathrm{IC}_{50}\right)$ for the 
Table 1. Scavenging Activities of 2-Amino-1,3-selenazoles 1 and Bis-[2amino-5-(1,3-selenazoyl)] Ketones 2 on the Superoxide Radical Anion

Inhibition (\%)
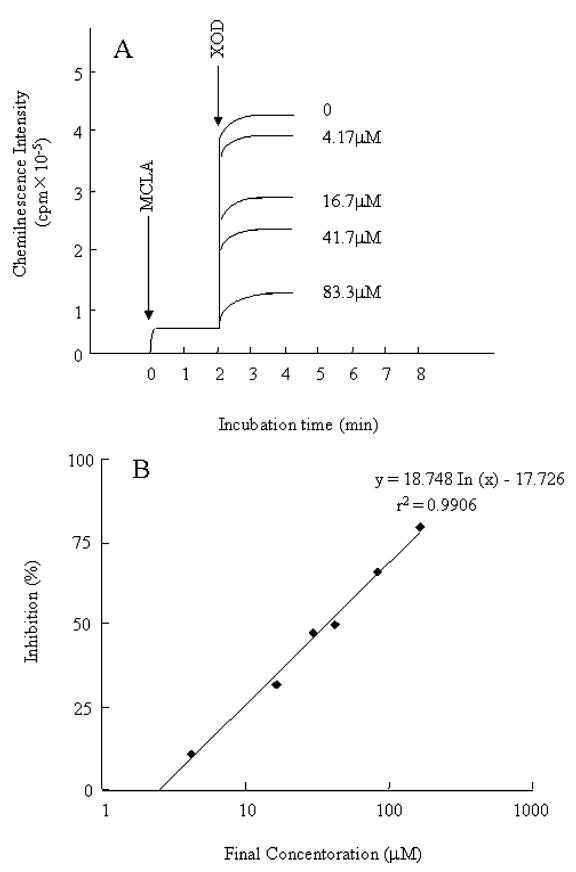

Fig. 1. Effect of Bis[2-dimethylamino-5-(1,3-selenazolyl)] Ketone 2a on 2-Methyl-6-(4-methoxyphenyl)-3,7-dihydroimidazo-[1,2-a]pyrazin-3-one Hydrochloride (MCLA)-Dependent Luminescence

(A) Chemiluminescence inhibition curves by compound 2a. Arrows indicate the time at which MCLA or xanthine oxidase (XOD) was added. (B) Fifty percent inhibitory concentrations $\left(\mathrm{IC}_{50}\right)$ for the compound.

compound 2a were $37.1 \mu \mathrm{m}$ (Fig. 1). We have systematically synthesized various organic selenium compounds including selenocarbamates, selenoureas, thioureas, and tertiary se-

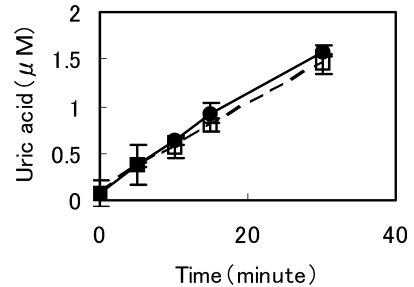

Fig. 2. Uric Acid Generation in Hypoxantine-Xanthine Oxidase System

--: Control, - $\square-$ : with 2a. Bis[2-dimethylamino-5-(1,3-selenazoly1)] ketone 2a of $83.3 \mu \mathrm{m}$ was added to the standard reaction mixture, and generation rate of uric acid was measured by uric acid measurement kit (L type WAKO UA - M; Wako Pure Chemical Industries, Ltd., Osaka, Japan). The data are representative of three separate experiments and shown by the means \pm S.E.

lenoamide compounds, and showed their SOSAs using a same chemiluminescence method. $\mathrm{IC}_{50}$ of these compounds were approximately a range of 0.1 to $100 \mu \mathrm{M} .{ }^{14-16)}$ Thus, SOSAs of newly synthesized compounds, bis-[5-(1,3-selenazolyl)] ketones $\mathbf{2 a}$ and $\mathbf{2 c}$ had similar SOSAs to that of previously synthesized other organic selenium compounds such as selenocarbamates and selenoureas. The results suggested that bis-(2-amino-5-selenazoyl) ketones $\mathbf{2}$ are useful organic selenium compounds as one of scavenger of ROSs. We investigated whether bis[2-dimethylamino-5-(1,3-selenazolyl)] ketone 2a could eliminate super oxide anion generated by XOD or not. For this purpose, the amounts of uric acid generation which is the by-product of superoxide anion in the standard reaction mixture with or without $\mathbf{2 a}$ were measured. The result was shown in Fig. 2. Generation of uric acid was not significantly affected by the addition of relatively high concentration of a compound $2 \mathrm{a}(83.3 \mu \mathrm{M})$. Thus, it is suggested that our compounds act as not inhibitor of XOD but scavenger of superoxide anion.

In conclusion, the present work shows the SOSAs of bis[2dimethylamino-5-(1,3-selenazolyl)] ketone 2a. Future studies will be directed toward elucidating the mechanism of action of the active compounds and toward synthesizing and studying additional analogues of bis[2-dimethylamino-5-(1,3-selenazolyl)] ketone $\mathbf{2 a}$.

Acknowledgements This work was supported by a Grant-in-Aid for Science Research from the Ministry of Education, Culture, Sports, Science and Technology of Japan (No. 15550030 and 17550099) to which we are grateful.

\section{REFERENCES}

1) Hsu P. C., Guo Y. L., Toxicology, 180, 33- 44 (2002).

2) Long J. F., Dutta P. K., Hogg B. D., Environ. Health Perspect., 105, 706-711 (1997).

3) Ramirez R., Carracedo J., Jimenez R., Canela A., Herrera E., Aljama P., Blasco M. A., J. Biol. Chem., 278, 836-842 (2003).

4) Fridovich I., Annu. Rev. Biochem., 64, 97-112 (1995).

5) Ricci J.-E., Gottlieb R. A., Green D. R., J. Cell. Biol., 160, 65-75 (2003).

6) Kimura H., Sawada T., Oshima S., Kozawa K., Ishioka T., Kato M., Curr. Drug Targets Imflamm. Allergy, 4, 489-495 (2005).

7) Kato M., Kimura H., Motegi Y., Tachibana A., Minakami H., Morikawa A., Kita H., J. Immunol., 169, 5252-5259 (2002).

8) Wendel A., Tiegs G., Biochem. Pharmacol., 35, 2115-2118 (1986).

9) Taino L., Fedeli D., Santroni A. M., Villarini M., Engman L., Falcioni G., Mutat. Res., 464, 269-277 (2000).

10) Jeong D. W., Kim T. S., Chung Y. W., Lee B. J., Kim I. Y., FEBS Lett., 517, 225-228 (2002). 
11) Engman L., Hallberg A., J. Org. Chem., 54, 2964-2966 (1989).

12) Zhang M., Nomura A., Uchida Y., Iijima H., Sakamoto T., Iishii Y., Morishima Y., Mochizuki M., Masuyama K., Hirano K., Sekizawa K., Free Radical Biol. Med., 32, $454-464$ (2002).

13) Nakamura Y., Feng Q., Kumagai T., Torikai K., Ohigashi H., Osawa T., Noguchi N., Niki E., Uchida K., J. Biol. Chem., 277, 2687-2694 (2002).

14) Takahashi H., Nishina A., Kimura H., Motoki K., Koketsu M., Ishi- hara H., Eur. J. Pharm. Sci., 23, 207-211 (2004).

15) Takahashi H., Nishina A., Fukumoto R., Kimura H., Koketsu M., Ishihara H., Life Sci., 76, 2185-2192 (2005).

16) Takahashi H., Nishina A., Fukumoto R., Kimura H., Koketsu M., Ishihara H., Eur. J. Pharm. Sci., 24, 291-295 (2005).

17) Koketsu M., Kogami M., Ando H., Ishihara H., Synthesis, 31-36 (2006).

18) Kimura H., Nakano M., FEBS Lett., 239, 347-350 (1988). 